\title{
THE IDENTITY OF WEAK AND STRONG EXTEN- SIONS OF DIFFERENTIAL OPERATORS
}

BY

K. O. FRIEDRICHS

In applying the theory of linear operators in Hilbert spaces or spaces $\mathfrak{R}_{p}$ to the solution of differential equation problems, it is impossible to retain the. meaning of differentiation in the ordinary sense; the concept of differential operator must be extended. Two such extensions offer themselves, a "weak" and a "strong" one. Existence theorems, when derived by variational methods, result most directly in terms of the weak extensions. It is the strong extension, however, which offers the natural approach to establishing properties of the solution; in particular those that lead to differentiability in the ordinary sense. The fact that both extensions are identical is therefore decisive.

The objective of this paper is to prove the identity of weak and strong extension for general linear differential operators. The main tool for the proof is a certain class of smoothing operators approximating unity, the "mollifiers." These mollifiers yield the identity of both extensions immediately for differential operators with constant coefficients; it is remarkable that they are a strong enough tool to yield this identity likewise for operators with non-constant coefficients.

While the present paper is not concerned with the application to existence problems $\left({ }^{1}\right)$, other miscellaneous applications and generalizations of the identity will be discussed.

In the main part of the paper ( $\$ \S 1-3)$ the identity of weak and strong extensions is proved with reference to the function space $\mathfrak{R}_{p}$. Generalization to function spaces enjoying a certain translation property is possible ( $\$ 5$ ). Under certain restrictions weak and strong extensions can be expressed in terms of adjointness (\$4). The miscellaneous applications $(\$ 6)$ refer in particular to underdetermined systems and to Haar's lemma.

1. Extended differential operators. Let $x=\left(x_{1}, \cdots, x_{m}\right)$ be a point in an $m$-dimensional Euclidean space. Let $R$ be an open region in that space. Let $u=\left\{u_{\sigma}\right\}, \sigma=1, \cdots, s$, be a system of functions defined in $R ; u=u(x)$ will in general simply be called a function. Let $A_{\mu}, \mu=1, \cdots, m, B$ be matrices transforming systems $u$ of $s$ functions into systems $A_{\mu} u, B u$ of $t$ functions.

Presented to the Society, February 26, 1944 ; received by the editors April 9, 1943.

(1) For elliptic differential equations the identity was used in an earlier paper [1]; for hyperbolic differential equations it can be used to solve Cauchy's initial problem [2]. Numbers in brackets refer to the bibliography at the end of the paper. 
The matrices $A_{\mu}$ and $B$ as functions of $x$ are to be continuous in $R$; in addition, $A_{\mu}$ is to possess continuous derivatives with respect to $x_{1}, \cdots, x_{m}$.

By $\mathfrak{E}$ and $\mathfrak{D}$ we denote the classes of functions $u(x)$ which are respectively continuous or continuously differentiable in $R$. The operator

$$
D_{\mu}=\partial / \partial x_{\mu}
$$

then transforms a function $u$ in $\mathfrak{D}$ into the function $D_{\mu} u$ in $\mathfrak{G}$. The differential operator to be considered in this paper is

$$
E=A_{\mu} D_{\mu}+B,
$$

summation with respect to $\mu$ from $\mu=1$ to $\mu=m$ being implied. The operator $E$ transforms a function $u$ in $\mathfrak{D}$ into a function in $\mathfrak{E}$,

$$
v=E u=A_{\mu} D_{\mu} u+B u .
$$

The "formal-adjoint" to $E$ is the operator

$$
E^{*}=-D_{\mu} A_{\mu}^{*}+B^{*}=-A_{\mu}^{*} D_{\mu}+B^{*}-D_{\mu} A_{\mu}^{*} \text {. }
$$

(the dot indicating that the operator $D_{\mu}$ does not apply beyond it). $A^{*}$ and $B^{*}$ are the transposed matrices of $A$ and $B$. By $u$ we denote functions to which there is a bounded closed subdomain $\dot{R}$ of $R$ outside of which $u=0$; by $\dot{\mathfrak{C}}$ or $\mathfrak{D}$ we denote the spaces of functions $\dot{u}$ in $\mathfrak{C}$ or $\mathfrak{D}$, respectively. For $u$ in $\mathfrak{E}, \dot{u}$ in $\mathfrak{\mathfrak { S }}$ we define the bilinear form

$$
(\dot{u}, u)_{R}=\int_{R} \dot{u} u d x, \quad d x=d x_{1} \cdots d x_{m},
$$

$\dot{u} u=\dot{u}_{\sigma} u_{\sigma}$ being the inner product of the vectors $\dot{u}$ and $u$. For $u$ in $\mathfrak{D}, \dot{u}$ in $\mathscr{D}$ we have the identity

$$
\left(E^{*} \dot{u}, u\right)_{R}=(\dot{u}, E u)_{R}
$$

Let $p$ be any number $p \geqq 1$. Then we define the modulus

$$
|u|=\left[\sum_{\sigma}|u|^{p}\right]^{1 / p}
$$

for functions $u=\left\{u_{\sigma}\right\}, \sigma=1, \cdots, s$, and further the norm

$$
\|u\|_{R}=\left[\int_{R}|u|^{p} d x\right]^{1 / p}
$$

We also admit $p=\infty$ and then set

$$
\begin{array}{rlr}
|u| & =\max _{\sigma}\left|u_{\sigma}\right|, & \sigma=1, \cdots, m, \\
\|u\|_{R} & =\max _{x}|u(x)|, & x \in R .
\end{array}
$$

The space $\dot{\mathfrak{C}}$ can be extended to spaces $\mathfrak{R}=\mathfrak{l}_{p}$, complete with respect to the 
norm \|\|$_{R}$, by either adjoining Lebesgue-integrable functions or by adjoining ideal elements (cf. Hausdorff, Mengenlehre, 2nd ed., 21.3, p. 106). (The space $\mathfrak{R}_{\infty}$ is here simply the space of continuous functions vanishing at the boundary of $R$.) Of course, we write $u=0$ if $\|u\|_{R}=0$ for $u$ in $\mathfrak{R}$. By $\mathbb{R}^{*}$ we denote the "adjoint" space $\mathfrak{R}^{*}=\mathfrak{R}_{p^{*}}$ with $1 / p^{*}+1 / p=1$. For $u$ in $\mathfrak{R}, u^{*}$ in $\mathfrak{l}^{*}$ the bilinear form

$$
\left(u^{*}, u\right)_{R}=\int_{R} u^{*} u d x
$$

can be defined and, as is well known,

$$
\left|\left(u^{*}, u\right)_{R}\right| \leqq\left\|u^{*}\right\|_{R}\|u\|_{R}
$$

We further note the well known

LEMMA 1.1. If $u$ in $\mathfrak{R}$ is such that $(\dot{u}, u)_{R}=0$ for all $\dot{u}$ in $\dot{D}$, then $u=0$.

In $\$ 2$ we shall obtain an incidental proof of it.

We now proceed to extend the operator $E$ to a subspace $\mathbb{B}$ of $\mathfrak{R}$, defined as follows:

The space $\$$ consists of all functions $u$ in $\mathbb{\&}$ to which there is a function $v$ in $\mathbb{R}$ such that

$$
\left(E^{*} \dot{u}, u\right)_{R}=(\dot{u}, v)_{R}
$$

holds for all $\dot{u}$ in $\dot{\mathfrak{D}}$.

It is clear that $\mathbb{B}$ contains $\mathfrak{D}$. Further $u=0$ implies $v=0$ by virtue of Lemma 1.1. Hence, the function $v$ is uniquely assigned to $u$ in $B$ and, consequently, $v$ is linear in $u$. Further, for $u$ in $\mathfrak{D}$, we have $v=E u$. Therefore, when we set

$$
v=E u \text { for } u \text { in }(S)
$$

the operation $E$, defined for $u$ in $(S)$, is an extension of the operator $E$ defined for $u$ in $\mathfrak{D}$; we term it the "weak" extension $\left({ }^{2}\right)$. For $u$ in $\&$ we then have (1.1) with every $\dot{u}$ in $\mathfrak{D}$.

To describe the "strong" extension we define:

The space $\mathfrak{F}$ consists of all functions $u$ in $\mathbb{R}$ to which there is a function $v$ in $\mathbb{B}$ and, to every proper $\left(^{3}\right)$ subregion $R^{\prime}$ of $R$, a sequence $u_{\epsilon}, \epsilon \rightarrow 0$, of functions in $\mathfrak{D}$ such that

$$
\left\|u_{\mathrm{c}}-u\right\|_{R^{\prime}} \rightarrow 0, \quad\left\|E u_{\mathrm{c}}-v\right\|_{R^{\prime}} \rightarrow 0, \quad \dot{\epsilon} \rightarrow 0 .
$$

(2) The operator $E$ in $\&$ could also be described as the adjoint of the formal adjoint $E^{*}$ in $\downarrow$ (cf. \$4).

(3) A "proper subregion $\boldsymbol{R}^{\prime}$ of $\boldsymbol{R}^{n}$ is meant to be an open region contained in a bounded closed subdomain of $\boldsymbol{R}$. 
Since relation (1.1) holds for $u=u_{\epsilon}$, relation (1.2) follows for all $\dot{u}$ with $R \subset R^{\prime}$; since $R^{\prime}$ was arbitrary, (1.2) holds for all $\dot{u}$ in $\dot{D}$. Hence $u$ is in (\$) and $v=E u$. Thus $\mathfrak{F}$ is a subspace of $\mathfrak{H}$. On the other hand, $\mathfrak{F}$ contains $\mathfrak{D}$. The operator $E$ defined in $\mathfrak{F}$ is therefore an extension of $E$ in $\mathfrak{D}$, the "strong" extension $\left({ }^{4}\right)$.

The goal of the present paper is the

MAIN TheOREM. $\mathfrak{F}=(\$)$.

Before proving the main theorem we should like to mention that generalizations of differential operators have been considered several times in the literature. M. Bôcher [3] has replaced the potential equation $\Delta u=f$ by $\int(\partial u / \partial n) d s=\iint f d x d y$ (in obvious notation). G. C. Evans [4] has generalized differential operators in a similar manner, in particular, the operator gradient $=\left\{D_{1}, \cdots, D_{m}\right\}[5]$. These generalizations and our weak extension are related. The class of functions, which equal one inside and zero outside, of rectangular cells plays the same rôle in Evans' generalization as the class of functions $\dot{u}$ in $\dot{D}$ in ours; instead of the left member in (1.2), an appropriate integral over the boundary of the cells occurs in Evans' definition and the resulting relation is to hold for almost all such cells. The identity of Evans' definition and the strong extension of the gradient was proved by C. B. Morrey [6] and J. W. Calkin [7]; the main tool for this proof was the operation of averaging over rectangular cells, of which our mollifiers are an analogue.

A generalization of second order differential equations which is essentially identical with the weak extension in our sense was employed by $\mathrm{N}$. Wiener [8] and by R. Courant [9]. The generalization introduced by D. C. Lewis [10] for nonlinear second order differential equations is similar to the strong extension. The weak and strong extensions for gradient and divergence were introduced and identified by the author [1] and applied to elliptic differential operators. Similar definitions for various geometrical operators were used by H. Weyl [11].

The extensions as proposed in the present paper offer several advantages. They are formulated solely in terms of the norm of the function spaces in which the extension is desired. No reference to the properties of the functions in relation to Lebesgue's theory is necessary. Also, it is not necessary to ascribe any meaning to the individual terms $D_{\mu} u$ in the extension of $A_{\mu} D_{\mu} u$,

It is true that the scope of our extension procedure is limited. For example, our procedure cannot be employed directly to extend differential operators into the space of bounded measurable functions $u(x)$ with the norm $\|u\|=$ true

(4) This strong extension has a certain weakness, in that relation (1.3) is required only for subdomains $\boldsymbol{R}^{\prime}$. If this relation were to hold for $\boldsymbol{R}$ instead of $\boldsymbol{R}^{\prime}$ ( $u_{\boldsymbol{\epsilon}}$ being in $\mathfrak{D}$ ), the extension could be described as the closure of $E$ in $\mathfrak{D}$; this closure coincides with the strong extension under certain conditions, cf. Theorem 4.2. 
maximum $|u(x)|$; for, the manifold of continuous functions is not dense in this space (cf., however, $\$ 5$, p. 28). However, in Hilbert spaces or spaces $\mathfrak{l}_{p}$, our method leads in a most direct way to the essential general properties of differential operators.

2. Integral operators. The proof of the main theorem evidently requires the construction, for every $u$ in $\mathfrak{S}$, of a sequence $u_{\epsilon}$ in $\mathfrak{D}$ such that relation (1.3) holds. This construction will be performed with the aid of certain integral operators.

It is convenient to formulate a few general properties of integral operators, which will be applied in two different cases.

By $k$ we denote matrices which transform functions $u$ into functions $k u$. Let $\boldsymbol{R}^{\prime}$ be a proper subregion of $\boldsymbol{R}$. Then we consider matrices $k=k\left(x^{\prime}, x\right)$ which are continuous functions of the pair $\left(x^{\prime}, \bar{x}\right)$ of points $x^{\prime}$ in $R, \bar{x}$ in $R$. We shall require property

PI. $k\left(x^{\prime}, \bar{x}\right)=0$ if $\bar{x}$ is outside of the cell $C_{\epsilon}\left(x^{\prime}\right):\left|x_{\mu}^{\prime}-\bar{x}_{\mu}\right|<\epsilon ; \mu=1, \cdots, m$; for $x^{\prime}$ in $R^{\prime}$.

The number $\epsilon$ is to be so small that all these cells are within a certain closed subdomain $\boldsymbol{R}_{\mathbf{0}}$; we sometimes emphasize property PI by setting $k=k_{\boldsymbol{\epsilon}}$.

We take $k\left(x^{\prime}, \bar{x}\right)$ as the kernel of an integral operator $K$, which transforms every function $u$ in $\mathbb{S}$ into the function

$$
K u\left(x^{\prime}\right)=\int_{R} k\left(x^{\prime}, \bar{x}\right) u(\bar{x}) d \bar{x} ;
$$

$K u$ is in $\mathcal{S}^{\prime}$ (that is, in the space ( 5 with reference to the subregion $R^{\prime}$ ). Clearly, there is a constant $C$ such that $\left|K u\left(x^{\prime}\right)\right| \leqq C\|u\|_{R}$. Consequently, the operator $K$ can be extended to functions in $\mathfrak{\&}$; the function $K u$ for $u$ in $\mathfrak{R}$ is also in $\mathfrak{C}^{\prime}$.

If $k\left(x^{\prime}, \bar{x}\right)$ possesses continuous derivatives $D_{\mu} k\left(x^{\prime}, \bar{x}\right)$ with respect to $x_{\mu}^{\prime}$, then $K u\left(x^{\prime}\right)$ is in $\mathfrak{D}^{\prime}$ for $u$ in $\mathbb{R}$.

The adjoint $K^{*}$ of $K$ is defined as the integral operator with the kernel $k^{*}\left(\bar{x}^{\prime}, x\right)$; here $k^{*}$ is the transposed of the matrix $k$. The operator $K^{*}$ transforms functions $u$ in $L^{\prime}$ into functions

$$
K^{*} u(x)=\int_{R^{\prime}} k^{*}\left(\bar{x}^{\prime}, x\right) u\left(\bar{x}^{\prime}\right) d \bar{x}^{\prime},
$$

which are in $\dot{夭}$. The operators $K$ and $K^{*}$ are related through the identity

$$
\left(K^{*} w, u\right)_{R}=(w, K u)_{R^{\prime}}, \quad \text { for } u \text { in } \mathbb{R}, w \text { in } \mathfrak{R}^{\prime} .
$$

Let $k_{\tau \sigma}, \sigma=1, \cdots, s, \tau=1, \cdots, t$, be the terms of the matrix $k$. We set

$$
|k|=\max _{\sigma, \tau}\left\{\sum_{\rho}\left|k_{\tau \rho}\right|, \sum_{\rho}\left|k_{\rho \sigma}\right|\right\} \text {. }
$$


Then we have the inequality

$$
|k u| \leqq|k||u| \text {. }
$$

It may be justified( $\left.{ }^{5}\right)$ to indicate its proof, which follows immediately from Hölders' inequality,

$$
\begin{aligned}
|k u|^{p} & =\sum_{\tau}\left|\sum_{\sigma} k_{\tau \sigma} u_{\sigma}\right|^{p} \leqq \sum_{\tau}\left[\sum_{\sigma}\left|k_{\tau \sigma}\right|^{1 / p^{*}}\left|k_{\tau \sigma}\right|^{1 / p}\left|u_{\sigma}\right|\right]^{p} \\
& \leqq \sum_{\tau}\left[\sum_{\sigma}\left|k_{\tau \sigma}\right|\right]^{p / p^{*}} \sum_{\sigma}\left|k_{\tau \sigma}\right|\left|u_{\sigma}\right|^{p} \leqq \sum_{\tau}|k|^{p / p^{*}} \sum_{\sigma}\left|k_{\tau \sigma}\right|\left|u_{\sigma}\right|^{p} \\
& \leqq|k|^{p / p^{*}}|k| \sum_{\sigma}\left|u_{\sigma}\right|^{p}=|k|^{p}|u|^{p} .
\end{aligned}
$$

We further introduce the "norm"

$$
\|K\|=\max _{x, x^{\prime}}\left\{\int_{R}|k(\bar{x}, x)| d \bar{x} ; \quad \int_{R}\left|k\left(x^{\prime}, \bar{x}\right)\right| d \bar{x}\right\}
$$

of the operator $K$. Then we have

$$
\|K u\| \leqq\|K\|\|u\| \text {. }
$$

This inequality is derived in the same manner as (2.2), first for functions $u$ in $\mathbb{E}$; it then carries over to $u$ in $\mathbb{R}$.

We now consider a sequence of operators $K_{\epsilon}, \epsilon \rightarrow 0$, enjoying property PI and further

PII. The norms $\left\|K_{\star}\right\|$ have a common bound $K_{0}$,

$$
\left\|K_{\bullet}\right\| \leqq K_{0}
$$

The matrices $k$ were so far assumed to transform systems of $s$ functions into systems of $t$ functions. All that has been stated, of course, also holds if $k$ is a square matrix, that is, transforms systems of $s$ (or $t$ ) functions into systems of $s$ (or $t$ ) functions. In case $k$ is a square matrix we further require property

PIII. There is a number $\kappa$ such that

$$
K_{\epsilon} 1=\kappa 1
$$

holds for $x^{\prime}$ in $R^{\prime}, 1$ being the unit matrix, that is,

$$
\int_{R} k_{\epsilon, \tau \sigma}\left(x^{\prime}, \bar{x}\right) d \bar{x}=\kappa \delta_{r \sigma}, \quad x^{\prime} \text { in } R^{\prime} .
$$

(5) (2.2) is not stated explicitly in Hardy, Littlewood, P6lya, Inequalities, but it is closely related to inequality 275 , p. 198 , which, however, is mentioned only as a very special case of a much more general inequality. 
When we do not assume $k$ to be a square matrix we require property

PIII $_{0}$. Identity

$$
K_{\epsilon} 1=0
$$

holds for $x^{\prime}$ in $R^{\prime}$.

From Property PIII we derive the relation

$$
\left\|K_{\epsilon} u-\kappa u\right\|_{R^{\prime}} \rightarrow 0
$$
as $\epsilon \rightarrow 0$;

from property PIII $_{0}$ we derive

$$
\left\|K_{\epsilon} u\right\|_{R^{\prime}} \rightarrow 0
$$
as $\epsilon \rightarrow 0$.

To do this we set $v_{\mathrm{e}}=K_{\mathrm{e}} u-\kappa u$ if $k_{\mathrm{e}}$ is a square matrix and PIII holds, and $v_{\epsilon}=K_{\epsilon} u$ if $\mathrm{PIII}_{0}$ holds. In both cases we find

$$
v_{\epsilon}\left(x^{\prime}\right)=\int_{R} k_{\epsilon}\left(x^{\prime} \bar{x}\right)\left[u(\bar{x})-u\left(x^{\prime}\right)\right] d \bar{x} .
$$

First we assume that $u(x)$ is in $\dot{\varepsilon}$ and let $\omega_{c}$ be the maximum of $\left|u(\bar{x})-u\left(x^{\prime}\right)\right|$ for $x^{\prime}$ in $R^{\prime}, x$ in the cell $\left|\bar{x}_{\mu}-x_{\mu}^{\prime}\right| \leqq \epsilon, \mu=1, \cdots, m$. Then, by virtue of (2.2) and PI, PII, we have

$$
\left|v_{\epsilon}\left(x^{\prime}\right)\right| \leqq\left\|K_{\epsilon}\right\| \omega_{\epsilon} \leqq K_{0} \omega_{\epsilon} .
$$

Due to the uniform continuity of $u(x)$ we have $\omega_{\epsilon} \rightarrow 0$ as $\epsilon \rightarrow 0$. Consequently, $v_{\epsilon}\left(x^{\prime}\right) \rightarrow 0$ as $\epsilon \rightarrow 0$ uniformly in $R^{\prime}$. Relations (2.6), (2.6) 0 thus follow for $u$ in غ.

If $u$ is any function in $\mathfrak{R}$ we approximate it by functions $u$ in $\dot{\mathfrak{E}}$ such that $\|u-\dot{u}\|_{R}$ is arbitrarily small. From

$$
\begin{aligned}
\left\|K_{\mathrm{e}} u-\kappa u\right\|_{R^{\prime}} & \leqq\left\|K_{\mathrm{e}}(u-\dot{u})\right\|_{R^{\prime}}+|\kappa|\|u-\dot{u}\|_{R^{\prime}}+\left\|K_{\mathrm{e}} \dot{u}-\kappa \dot{u}\right\|_{R^{\prime}} \\
& \leqq\left(K_{0}+|\kappa|\right)\|u-\dot{u}\|_{R}+\left\|K_{\mathrm{e}} \dot{u}-\kappa \dot{u}\right\|_{R^{\prime}}
\end{aligned}
$$

we see that (2.6) holds for any $u$ in $\mathfrak{R}$, if PIII holds. The same is, of course, true for $(2.6)_{0}$ if $\mathrm{PIII}_{0}$ holds.

We now construct the mollifiers as a special sequence of operators $K_{\epsilon}=J_{\epsilon}$. Let $j(\xi)$ be a function of the variable number $\xi$ possessing derivatives of all orders and satisfying the relations

$$
\begin{gathered}
j(\xi) \geqq 0, \quad j(\xi)=0 \quad \text { for }|\xi| \geqq 1, \\
\int_{-1}^{1} j(\xi) d \xi=1 .
\end{gathered}
$$

Then we set, for $\epsilon>0$,

$$
j_{\epsilon}\left(x^{\prime}-\bar{x}\right)=\epsilon^{-m} j\left(\epsilon^{-1}\left(x^{\prime}-\bar{x}\right)\right) \cdots j\left(\epsilon^{-1}\left(x_{m}^{\prime}-\bar{x}_{m}\right)\right) \cdot 1 .
$$

(Here 1 is the unit matrix of order either $s$ or $t$.) Clearly $j_{\text {c }}$ enjoys property PI 
and can be taken as kernel of an integral operator $J_{e}$. That $J_{e}$ enjoys also PII with $K_{0}=1$ and PIII with $\kappa=1$ follows immediately from the definition of $j(\xi)$. Consequently, we have from (2.4), (2.3), and (2.6) the relations (cf. [12,] for $m=1$ )

$$
\begin{aligned}
\left\|J_{\epsilon}\right\| & \leqq 1, \\
\left\|J_{\epsilon} u\right\|_{R^{\prime}} & \leqq\|u\|_{R^{\prime},} \\
\left\|J_{\epsilon} u-u\right\|_{R^{\prime}} & \rightarrow 0
\end{aligned}
$$$$
\text { as } \epsilon \rightarrow 0 \text {. }
$$

We mention incidentally that relation (2.9) yields a proof of Lemma 1.1. We need only consider the matrix $j_{\epsilon}\left(x^{\prime}-\bar{x}\right)$ for fixed $x^{\prime}$ as a system of functions in $\mathfrak{D}$. The assumption of Lemma 1.1 then leads to $J_{\mathrm{e}} u\left(x^{\prime}\right)=0$. Consequently (2.9) yields $\|u\|_{R^{\prime}}=0$. Since $R^{\prime}$ was arbitrary we have $\|u\|_{R}=0$.

The main application of the mollifiers, however, is that they furnish in

$$
u_{\epsilon}=J_{\epsilon} u,
$$

for $u$ in $\mathbb{S}$, an approximating sequence as required for the strong extension.

3. Proof of the main theorem. The proof of the main theorem rests on the following basic identity,

$$
J_{\epsilon} E-E J_{\epsilon}=\left[\bar{D}_{\mu}\left(A_{\mu}^{\prime}-\bar{A}_{\mu}\right)-\left(B^{\prime}-\bar{B}\right)\right] J_{\epsilon} \cdot,
$$

which holds when applied in functions in $\$$, that is, in the space in which the weak extension of $E$ is defined. The right member is the integral operator with the kernel

$$
\left[\bar{D}_{\mu}\left(A_{\mu}^{\prime}-\bar{A}_{\mu}\right)-\left(B^{\prime}-\bar{B}\right)\right] j_{c}\left(x^{\prime}-\bar{x}\right)
$$

where

$$
A_{\mu}^{\prime}=A_{\mu}\left(x^{\prime}\right), \quad \bar{A}_{\mu}=A_{\mu}(\bar{x}), \quad B^{\prime}=B\left(x^{\prime}\right), \quad \bar{B}=B(\bar{x}), \quad \bar{D}_{\mu}=\partial / \partial \bar{x}_{\mu} .
$$

To derive identity (3.1) we first note that $E J_{c}$ is an integral operator with the kernel

$$
\left(A_{\mu}^{\prime} D_{\mu}^{\prime}+B^{\prime}\right) j_{\epsilon}\left(x^{\prime}-\bar{x}\right) ;
$$

using the relation

$$
D^{\prime} j_{\epsilon}\left(x^{\prime}-\bar{x}\right)=-\bar{D} j_{\epsilon}\left(x^{\prime}-\bar{x}\right)
$$

we find

$$
E J_{\mathbf{c}}=\left(-\bar{D}_{\mu} A_{\mu}^{\prime}+B^{\prime}\right) J_{\epsilon} \cdot .
$$

We proceed to show that for $u$ in $\mathbb{B}$

$$
J_{\epsilon} E=\left(-\bar{D}_{\mu} \bar{A}_{\mu}+\bar{B}\right) J_{\epsilon} \bullet
$$

holds. To this end we begin with the relation 


$$
\left(w, J_{\epsilon} E u\right)_{R^{\prime}}=\left(J_{\epsilon}^{*} w, E u\right)_{R}
$$

which is valid by virtue of $(2.1)$ for $w$ in $\mathfrak{R}^{\prime}, u$ in $\mathfrak{R}^{\prime}$. We now make use of the fact that relation (1.1) holds for $u$ in $\mathfrak{S}, \dot{u}$ in $\dot{\mathfrak{D}}$. Since $J_{e}^{*} w$ is evidently in $\dot{\mathfrak{D}}$, for $w$ in $\dot{\mathfrak{D}}$, we obtain

$$
\left(J_{\epsilon}^{*} w, E u\right)_{R}=\left(E^{*} J_{\bullet}^{*} w, u\right)_{R} .
$$

The operator $E^{*} J_{\epsilon}^{*}$ is an integral operator with the kernel $-\left(D_{\mu} A_{\mu}{ }^{*}\right.$ $\left.-B^{*}\right) j_{\epsilon}\left(\bar{x}^{\prime}-x\right)$; it is the adjoint of the operator $-\left(\bar{D}_{\mu} \bar{A}_{\mu}-\bar{B}\right) J_{\epsilon} \cdot$ with the kernel $-\left(\bar{D}_{\mu} \bar{A}_{\mu}-\bar{B}\right) j_{\epsilon}\left(x^{\prime}-\bar{x}\right)$, which is of the type $K_{\epsilon}$ considered in $\S 2$. Applying identity (2.1) to this operator we obtain from (3.4), (3.5)

$$
\left(w, J_{\epsilon} E u\right)_{R^{\prime}}=-\left(w,\left(\bar{D}_{\mu} \bar{A}_{\mu}-\bar{B}\right) J_{\epsilon} \cdot u\right)_{R^{\prime}}
$$

Since $w$ is arbitrary, relation (3.3) follows, cf. Lemma 1.1. Subtraction of (3.2) and (3.3) yields relation (3.1).

Let us first suppose that the matrices $A_{\mu}$ and $B$ are constant. Then the right member in relation (3.1) vanishes and we have

$$
J_{\epsilon} E=E J_{\epsilon}
$$

for $u$ in $(\mathcal{G}$. Relation (2.9) applied to $E u, u$ in $(\mathcal{G}$, now gives

$$
\left\|E J_{\epsilon} u-E u\right\|_{R^{\prime}} \rightarrow 0 \quad \text { as } \epsilon \rightarrow 0,
$$

which together with $\left\|J_{\odot} u-u\right\|_{R^{\prime} \rightarrow 0}$ shows that $u$ is in $\mathfrak{F}$ and thus proves the main theorem.

If the matrices $A_{\mu}$ and $B$ are not all constant we have, instead of (3.6),

$$
\left\|J_{\epsilon} E u-E J_{\epsilon} u\right\|_{R^{\prime}} \rightarrow 0
$$

for $u$ in $B$. To prove this we first estimate

$$
\left\|\left(B^{\prime}-B\right) J_{\epsilon} \cdot u\right\|_{R^{\prime}} \leqq \beta_{\epsilon}\left\|J_{\epsilon} u\right\|_{R^{\prime}} \leqq \beta_{\epsilon}\|u\|_{R},
$$

where $\beta_{\epsilon}$ is the l.u.b. of

$$
\left|B\left(x^{\prime}\right)-B(\bar{x})\right|
$$

for $x^{\prime}$ in $R^{\prime}, \bar{x}$ in the cell $\left|x_{\mu}^{\prime}-\bar{x}_{\mu}\right| \leqq \epsilon, \mu=1, \cdots, m$. Clearly $\beta_{\epsilon} \rightarrow 0$ as $\epsilon \rightarrow 0$, since $B(x)$ was assumed to be continuous. Thus

$$
\left\|\left(B^{\prime}-B\right) J_{\epsilon} \cdot u\right\|_{R^{\prime}} \rightarrow 0 .
$$

Secondly we prove

$$
\left\|\bar{D}_{\mu}\left(A_{\mu}^{\prime}-\bar{A}_{\mu}\right) J_{\epsilon} \cdot u\right\|_{R^{\prime}} \rightarrow 0
$$

as $\epsilon \rightarrow 0$.

This relation will follow from the fact that the integral operator $H_{\epsilon}=\bar{D}_{\mu}\left(A_{\mu}^{\prime}-\bar{A}_{\mu}\right) J_{\epsilon} \cdot$ enjoys the properties PI, PII, PIII 0 . Property PI is 
obvious. Property $\mathrm{PIII}_{0}$ follows from the fact that the kernel $h_{\mathrm{a}}$ of $H_{\mathrm{a}}$ happens to be of the form

where

$$
h_{\epsilon}\left(x^{\prime}, \bar{x}\right)=\bar{D}_{\mu} \phi_{\mu}(\bar{x})
$$

$$
\phi_{\mu}(\bar{x})=\left(A_{\mu}^{\prime}-\bar{A}_{\mu}\right) j_{\epsilon}\left(x^{\prime}-\bar{x}\right),
$$

considered a function of $\bar{x}$, is in $\dot{D}$ when $x^{\prime}$ is in $R^{\prime}$. For such functions $\phi_{\mu}$ in $\mathfrak{D}$ obviously

$$
\int_{R} \bar{D}_{\mu} \phi_{\mu}(\bar{x}) d \bar{x}=0 .
$$

To deduce property PII for $H_{\mathrm{c}}$ we split

$$
H_{\epsilon}=\bar{D}_{\mu}\left(A_{\mu}^{\prime}-\bar{A}_{\mu}\right) J_{\epsilon} \cdot=-\bar{D}_{\mu} \bar{A}_{\mu} \cdot J_{\epsilon}+\left(A_{\mu}^{\prime}-\bar{A}_{\mu}\right) D_{\mu} J_{\epsilon} \cdot
$$

Using (2.7) we find

$$
\left\|\bar{D}_{\mu} \bar{A}_{\mu} \cdot J_{\epsilon}\right\| \leqq \gamma
$$

where $\gamma$ is the maximum of $\left|D_{\mu} A_{\mu}(x)\right|$ for $x$ in the domain $\dot{R}$ outside of which

$$
j_{\epsilon}\left(x^{\prime}-x\right)=0
$$
when $x^{\prime}$ is in $R$.

Further we have

$$
\left\|\left(A_{\mu}^{\prime}-\bar{A}_{\mu}\right) \bar{D}_{\mu} J_{\epsilon} \cdot\right\| \leqq \eta \epsilon \sum_{\mu}\left\|\bar{D}_{\mu} J_{\epsilon}\right\|,
$$

where $\eta$ is the maximum of

$$
\sum_{\rho}\left|D_{\rho} A_{\mu}(x)\right|
$$$$
\mu=1, \cdots, m,
$$

for $x$ in $\dot{R}$. One easily verifies that

$$
\epsilon\left\|\bar{D}_{\mu} J\right\|=\int_{-1}^{1}\left|j^{\prime}(\xi)\right| d \xi=\zeta
$$

is independent of $\epsilon$. Hence we obtain

$$
\left.\left\|\bar{D}_{\mu}\left(A_{\mu}^{\prime}-\bar{A}_{\mu}\right) J_{\epsilon} \cdot\right\| \leqq \gamma+m \eta \zeta\right)
$$

which is independent of $\epsilon$. Thus property PII is established for $H_{\epsilon}$. Relation (3.10) is then a consequence of $(2.6)_{0}$ applied to $K_{\mathrm{e}}=H_{\mathrm{e}}$.

Addition of (3.9) and (3.10) yields (3.8) for $u$ in $\&$ by virtue of identity (3.1). We now see that for $u$ in \&S the sequence $u_{\epsilon}=J_{\epsilon} u$ satisfies

$$
\left\|E u_{\epsilon}-E u\right\|_{R^{\prime}} \leqq\left\|E J_{\epsilon} u-J_{\epsilon} E u\right\|_{R^{\prime}}+\left\|J_{\epsilon} E u-E u\right\|_{R^{\prime}} \rightarrow 0
$$

as $\epsilon \rightarrow 0$, in view of (3.8) and (2.9) applied to $E u$. Since also $\left\|u_{\epsilon}-u\right\|_{R^{\prime}} \rightarrow 0$, it is shown that $u$ is in $\mathfrak{F}$. Thus the main theorem is proved. 
4. Adjoint operators. We recall some familiar notions: The operator $H$ defined in a space $B \subset R$ is closed if

$$
\left\|u_{\mathrm{e}}-u\right\|_{R} \rightarrow 0, \quad\left\|H u_{\mathrm{e}}-v\right\|_{R} \rightarrow 0, \quad \epsilon \rightarrow 0,
$$

for $u_{\mathfrak{e}}$ in $\mathfrak{P}, u, v$ in $\mathfrak{R}$ implies $u$ in $\mathfrak{B}, H u=v$. Clearly the operator $E$ in $\mathfrak{B}=\mathfrak{F}$ is closed, while $E$ in $\dot{D}$ is not.

The operator $H$ in $\mathfrak{B}$ is the closure of $\mathfrak{B}^{\prime} \subset \mathfrak{P}$ if to every $u$ in $\mathfrak{P}$ there is a sequence $u_{c}$ in $\mathfrak{P}^{\prime}$ such that

$$
\left\|u_{\mathrm{e}}-u\right\|_{R} \rightarrow 0, \quad\left\|H u_{\mathrm{e}}-H u\right\|_{R} \rightarrow 0,
$$$$
\epsilon \rightarrow 0 \text {. }
$$

Let $\mathfrak{F}_{\infty}$ consist of all $u$ in $\mathfrak{F}$ to which there is a sequence $u_{e}$ in $\mathfrak{D}$ such that

$$
\left\|u_{e}-u\right\|_{R} \rightarrow 0, \quad\left\|E u_{e}-E u\right\|_{R} \rightarrow 0 \text {, }
$$$$
\epsilon \rightarrow 0 \text {. }
$$

Then $E$ in $\mathfrak{F}_{\infty}$ is the closure of $E$ in $\mathfrak{D} ;$ in general $\mathfrak{F}_{\infty} \neq \mathfrak{F}$. One observes that the difference of the definition of $\mathfrak{F}$ and $\mathfrak{F}_{\infty}$ lies in the fact that relation (4.1) refers to the total region $R$ and not only to subregion $\boldsymbol{R}^{\prime}$. The condition for a function in $\mathfrak{F}$ to belong to $\mathfrak{F}_{\infty}$ can be considered a boundary condition (cf. $[1$, p. 534 and 9, p. 481]).

The operator $H^{*}$ in $\mathfrak{B}^{*} \subset \mathbb{R}^{*}$ is the adjoint to $H$ in $\mathfrak{B}$ if

$$
\left(u^{*}, H u\right)_{R}=\left(v^{*}, u\right)_{R}
$$

for $u^{*}, v^{*}$ in $\mathfrak{R}^{*}$, and all $u$ in $\mathfrak{B}$, implies $u^{*}$ in $\mathfrak{B}^{*}$ and $H^{*} u^{*}=v^{*}$. An adjoint operator is always closed. Clearly, the operator $E$ in $B=\mathfrak{F} \subset \mathfrak{R}$ is the adjoint to $E^{*}$ in $\mathfrak{D}$ and hence to $E^{*}$ in $\mathfrak{F}_{\infty}^{*}$, as is seen from the definition of $\mathfrak{H}$. The question arises whether $E$ in $\mathfrak{F}_{\infty} \subset \mathfrak{R}$ is the adjoint to $E^{*}$ in $\mathfrak{F}^{*}$. Let us denote by $\mathfrak{S}_{\infty}$ the space of all $u$ in $\mathfrak{F}$ for which

$$
\left(E^{*} u^{*}, u\right)_{R}=\left(u^{*}, u\right)_{R}
$$

holds with all $u^{*}$ in $\mathfrak{F}^{*}$. Then $E$ in $\mathfrak{S}_{\infty} \subset \mathfrak{Q}$ is the adjoint of $E^{*}$ in $\mathfrak{F}^{*}$. Our aim is to investigate whether or not $\mathbb{S}_{\infty}=\mathfrak{F}_{\infty}$ is true.

In case $\mathfrak{l}=\mathfrak{R}_{2}$ is a Hilbert space, $\mathfrak{S}_{\infty}=\mathfrak{F}_{\infty}$ follows from $\mathfrak{F}^{*}=\mathfrak{F}^{*}$ by virtue of the projection theorem, as von Neumann [13] had discovered. For the special operator gradient, which transforms $u$ into grad $u=\left\{D_{1} u, \cdots, D_{m} u\right\}$, it could be proved to be true for spaces $\mathbb{R}=\ell_{p}$ using a result of Morrey [14a]. For our general differential operators this reciprocity obtains at least if the coefficients $A, B$, and the region $R$ satisfy restrictive conditions. We formulate very strong restrictive conditions.

We term a region contractible if to every $\epsilon>0$ there is a one-to-one mapping $x^{\prime}=T_{e}(x)$ of $R$ onto a proper subregion of $R$, satisfying the following conditions:

1. The cells $C_{\epsilon}\left(T_{c}(x)\right)$ are in a bounded closed subdomain of $R$ when $x$ is in $R$.

2. $T_{\mathrm{c}}(x)$ possesses continuous second derivatives. 
3. $\epsilon^{-1}\left|T_{\epsilon}(x)-x\right|$ is bounded, uniformly in $x$ and $\epsilon$.

4. With the notation

$$
S_{\epsilon}(x)=\left\{S_{\epsilon, \mu \nu}(x)\right\}=D_{\mu} T_{\epsilon, \nu}(x),
$$

$\epsilon^{-1}\left|S_{\epsilon}(x)-1\right|$ is bounded, uniformly in $x$ and $\epsilon$.

5. $\left|D_{\mu} S_{\epsilon, \mu \nu}\right|$ is uniformly bounded and approaches zero in every bounded closed subdomain of $\boldsymbol{R}$.

We then state

THEOREM 4.1. The operator $E$ in $\mathfrak{F}_{\infty}$ is the adjoint to $E^{*}$ in $\mathfrak{F}^{*}$, that is, $\mathfrak{B}_{\infty}=\mathfrak{F}_{\infty}$ under the following conditions:

(I) the region $R$ is contractible.

(II) $B(x), A_{\mu}(x)$ and the derivatives $D_{\mu} A_{\mu}(x)$ are bounded in $R$.

For the proof we change the definition of the mollifiers slightly by taking

$$
j_{\epsilon}\left(x-T_{\epsilon}(\bar{x})\right)
$$

for their kernels. Then $J_{\iota} u$ is in $\mathfrak{D}$ in view of condition 1. By virtue of condition 4 we have that $\left\|J_{\epsilon} 1\right\|$ is bounded and $\left\|J_{\epsilon} 1-1\right\|_{R^{\prime}} \rightarrow 0$ as $\epsilon \rightarrow 0$ for every bounded closed subdomain $R^{\prime}$. These properties, instead of $J_{\mathbf{l}} 1=1$, are sufficient to insure

$$
\left\|J_{\epsilon} u-u\right\|_{R} \rightarrow 0, \quad \epsilon \rightarrow 0 \text { for } u \text { in } \mathbb{R} .
$$

The function $J_{e}^{*} w$, for $w$ in $\dot{\mathfrak{D}}$, need not be in $\mathfrak{D}$, but it is in $\mathfrak{D}$, and by virtue of assumption (1.1) $J_{e}^{*} w$ is in $\mathfrak{F}^{*}$. Consequently, (3.5) remains valid for $u$ in $\oiint_{\infty}$ in view of (4.2). Therefore also (3.3), that is,

$$
J_{\epsilon} E=-\overline{D_{\mu} A_{\mu}} J_{\epsilon} \cdot \bar{B} J_{\epsilon},
$$

remains valid for $u$ in $\mathcal{S}_{\infty}$. This relation is equivalent with

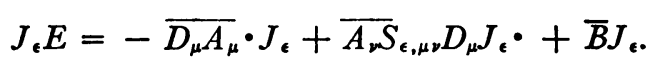

From

$$
E J_{\epsilon}=A_{\mu} D_{\mu} J_{\epsilon}+B J_{\epsilon}
$$

we obtain, instead of (3.2), the relation

$$
E J_{\epsilon}=A_{\mu} \bar{D}_{\nu}{\overline{S_{\epsilon, \mu \nu}}}_{-1}^{-1} J_{\epsilon}-\bar{D}_{\nu} A_{\mu} \bar{S}_{\epsilon, \mu \nu}^{-1} J_{\epsilon} \cdot+B_{\epsilon} J .
$$

From (4.5) and (4.6) together with properties 2, 3, 4 it follows that $\left\|E J_{e}-J_{e} E\right\|$ is bounded. From (4.4) and (4.7) together with properties 5 and 4 then follows

$$
\left\|E J_{\epsilon} u-J_{\epsilon} E u\right\|_{R} \rightarrow 0
$$$$
\text { as } \epsilon \rightarrow 0
$$

for $u$ in $\mathfrak{S}_{\infty}$. Hence $\left\|E J_{\epsilon} u-E u\right\|_{R} \rightarrow 0$. Thus Theorem 4.1 is proved. 
We mention incidentally

Theorem 4.2. Assume the conditions (I), (II) of Theorem 4.1 to be valid. Then to every $u$ in $\mathfrak{F}$ there is a sequence $u_{\mathfrak{\epsilon}}$ in $\mathfrak{D}$ such that

$$
\left\|u_{\epsilon}-u\right\|_{R} \rightarrow 0, \quad\left\|E u_{\epsilon}-E u\right\|_{R} \rightarrow 0 .
$$

That is, the restriction to subregions $R^{\prime}$ in relation (1.3), defining the strong extension, can be omitted.

To prove the statement one need only set $u_{\epsilon}=J_{e}^{*} u, J_{\epsilon}$ being the operator as introduced in this section. Otherwise the proof is similar to that of Theorem 4.1 (by the way, property 5 is not even used). We refrain from carrying it through.

5. Generalized norms. It may be of interest to note that the main theorem holds with reference to a more general type of norms. The norms we have in mind are characterized by the following properties:

I. To every subregion $S$ of $R$, the norm $\|u\|_{S}$ is defined such that

$$
\|u\|_{S_{1}} \leqq\|u\|_{S_{2}} \quad \text { if } S_{1} \subset S_{2} .
$$

II. Let $u_{a}(x)=u(x-a), a$ being an $m$-dimensional vector; denote by $S+a$ the region of all $x$ for which $x-a$ is in $S$. Assume that $S$ and $S+a$ are in $R$. Then

$$
\left\|u_{a}\right\|_{s+a}=\|u\|_{s}
$$

that is, the norm is invariant under translation.

III. Let $q(x)=\left\{q_{\tau \sigma}(x)\right\}$ be a continuous matrix, let, as before, $|q(x)|$ be the maximum of

$$
\sum_{\rho}\left|q_{\rho \sigma}(x)\right|, \quad \sum_{\rho}\left|q_{\tau \rho}(x)\right|, \quad \sigma=1, \cdots, s ; \tau=1, \cdots, t,
$$

and set

$$
\langle q\rangle_{s}=\underset{x}{\text { l.u.b. }}|g(x)| \text {, }
$$
$x$ in $\mathbf{S}$.

Then

$$
\|g u\|_{s} \leqq\langle q\rangle_{s}\|u\|_{s}
$$

Of course we require the standard properties of a norm, as, for example, the triangular inequality

$$
\left\|\alpha_{1} u_{1}+\alpha_{2} u_{2}|| \leqq\left|\alpha_{1}\right|\right\| u_{1}||+\left|\alpha_{2}\right| \| u_{2}||
$$

We then introduce a space $\mathfrak{R}$ which with respect to the norm $\|u\|$ is complete and contains $\dot{\delta}$ densely. The latter condition entails property

IV. For every proper subregion $R^{\prime}$ of $R$, 


$$
\left\|u_{a}-u\right\|_{R^{\prime}} \rightarrow 0
$$

It is clear that the norm

$$
\|u\|_{S}=N S_{S}^{p}(u)=\left[\int_{S}|u|^{p} d x\right]^{1 / p}
$$

belonging to the spaces $\mathbb{R}=\mathfrak{R}_{p}$ enjoys the enumerated properties. Relation (5.3) in particular follows immediately from (2.3). The norm $N^{p}$ may be generalized as follows. With

$$
\left|x^{\prime}-x\right|=\left[\left(x_{1}^{\prime}-x_{1}\right)^{2}+\cdots+\left(x_{m}^{\prime}-x_{m}\right)^{2}\right]^{i / 2}
$$

and a number $\alpha<m$, we set

$$
\|u\|=N_{S}^{p, \alpha}(u)=\underset{x^{\prime}}{\text { l.u.b. }}\left[\int_{S}|u(x)|^{p}\left|x^{\prime}-x\right|^{-\alpha} d x\right]^{1 / p},
$$

for $x^{\prime}$ in $S$ (that is, the l.u.b. refers to $x^{\prime}$ in $S$ ). Properties I and II are obvious from this definition, property III again follows from (2.3).

A different generalization, the "Morrey norm" $\left.{ }^{(}\right)$can be defined as follows: Let $S_{x^{\prime}, r}$ be the common part of $S$ and the circle $\left|x-x^{\prime}\right|<\epsilon, x^{\prime}$ being a point in $S$. Then, with $\beta \leqq m$, we set

$$
\|u\|_{S}=M_{S}^{p, \beta}(u)=\underset{x}{\text { l.u.b. }} \underset{r}{\text { l.u.b. }}\left[r^{-\beta} \int_{S_{x^{\prime}, r}}|u(x)|^{p} d x\right]^{1 / p},
$$

for $x^{\prime}$ in $S, r>0$.

Property III will be valid if the space $\mathfrak{R}$ is defined as the closure of $\dot{\mathfrak{C}}$. There is, however, another possibility of defining a space $\mathfrak{M}$, which is complete with respect to $M_{R}^{p, \beta}$, namely, as the space of all $u$ in $\Omega_{p}$ for which $M_{R}^{p, \beta}(u)$ is finite. This space $\mathfrak{M}$ does not enjoy property IV (for $\beta>0$ ), as can be seen by obvious counter-examples. For $\beta=m$, in particular, we have $M_{R}^{p, m}(u)=\langle u\rangle_{R}$. In this case, therefore, $\mathfrak{R}$ is the space of continuous functions vanishing at the boundary of $R$, while $\mathfrak{M}$ is equivalent to the class of essentially bounded measurable functions, which is known not to contain $\dot{S}$ densely. For such spaces $\mathfrak{M}$, therefore, our main theorem does not hold, unless the definition of the strong extension is modified by substituting $N_{R}^{p}$ for $M_{R}^{p, \beta}$ in (1.3).

Finally we mention that the Hölder norm

$$
\text { l.u.b. }\left|x_{1}-x_{2}\right|^{-\alpha}\left|u\left(x_{1}\right)-u\left(x_{2}\right)\right|, x_{1}, x_{2} \text { in } S \text {, }
$$

is not covered by the theory of the present section.

We first consider integral operators of a special kind.

( $\left.{ }^{6}\right)$ Morrey [14] had used the condition that $M^{p, \beta}(u)$ be finite (for $p=2, m=2, \beta<1$ ) and discovered that it is the clue to a treatment of nonquadratic minimum problems. 
Let $R^{\prime}$ be a proper subregion of $R$. Choose $\epsilon$ so small that the cell $C_{\epsilon}\left(x^{\prime}\right)$, $\left|x_{r}^{\prime}-x_{r}\right| \leqq \epsilon, r=1, \cdots, m$, is in $R$ when $x^{\prime}$ is in $R^{\prime}$.

Let $h_{\epsilon}(y)$ be a continuous function which vanishes outside of the cell $C_{\epsilon}$, $\left|y_{\mu}\right| \leqq \epsilon, \mu=1, \cdots, m$. Set

$$
\left\|H_{\epsilon}\right\|=\int_{C_{\epsilon}}\left|h_{\epsilon}(y)\right| d y .
$$

Then we consider the integral operation $H_{\epsilon}$ which transforms the functions $u$ in $\mathfrak{R}$ into functions

$$
H_{\epsilon} u\left(x^{\prime}\right)=\int_{R} h_{\epsilon}\left(\bar{x}-x^{\prime}\right) u(\bar{x}) d \bar{x}
$$

defined for $x^{\prime}$ in $R$. Clearly $H_{\epsilon}$ is applicable to any $u$ in $\mathfrak{R}$, and $H_{\epsilon} u$ is in $\mathcal{C}^{\prime}$. Moreover, we have

$$
\left\|H_{\iota} u\right\|_{R^{\prime}} \leqq\left\|H_{\epsilon}\right\|\|u\| \text {. }
$$

To prove this inequality we first observe that $H_{\mathrm{e}} u$ can be written in the form

$$
H_{\epsilon} u\left(x^{\prime}\right)=\int_{C_{\epsilon}} h_{\epsilon}(y) u_{y}\left(x^{\prime}\right) d x^{\prime},
$$

$u_{y}\left(x^{\prime}\right)=u\left(x^{\prime}-y\right)$ being defined for $x^{\prime}$ in $R^{\prime}$ since $\epsilon$ was so chosen that $R^{\prime}-y$ lies within $R$. Expression (5.6) for $u$ in $\dot{\mathcal{S}}$ can be uniformly approximated by a sum

$$
\alpha u_{a}\left(x^{\prime}\right)+\beta u_{b}\left(x^{\prime}\right)+\cdots,
$$

the vectors $a, b, \ldots$ being in $C_{\epsilon}$. The triangular inequality applied to this sum yields

$$
\begin{aligned}
\left\|\alpha u_{a}+\beta u_{b}+\cdots\right\|_{R^{\prime}} & \leqq|\alpha|\left\|u_{a}\right\|_{R^{\prime}}+|\beta|\left\|u_{b}\right\|_{R^{\prime}}+\cdots \\
& =|\alpha|\|u\|_{R^{\prime}+a}+|\beta|\|u\|_{R^{\prime}-b}+\cdots \\
& \leqq\{|\alpha|+|\beta|+\cdots\}\|u\|_{R} .
\end{aligned}
$$

An obvious limit process then leads to the desired inequality (5.5) for $u$ in $\dot{\mathfrak{S}}$. Clearly it can be extended to $u$ in $\&$.

The analogue of relation (2.6) for operators $H_{\epsilon}$ is

$$
\left\|H_{\epsilon} u-\kappa u\right\|_{R^{\prime}} \rightarrow 0
$$

if

$$
\kappa=\int_{C_{\epsilon}} h_{\epsilon}(y) d y
$$


is independent of $\epsilon$ and $\left\|H_{\mathrm{\epsilon}}\right\| \leqq H_{0}$ is bounded. To prove this relation we observe

$$
H_{\epsilon} u\left(x^{\prime}\right)-\kappa u\left(x^{\prime}\right)=\int_{C_{\epsilon}} h_{\epsilon}(y)\left[u_{y}\left(x^{\prime}\right)-u\left(x^{\prime}\right)\right] d y,
$$

whence, as for the proof of (5.5),

$$
\left\|H_{\mathrm{e}} u-\kappa u\right\|_{R^{\prime}} \leqq\left\|H_{\mathrm{e}}\right\|\left\|u_{y}-u\right\|_{\dot{R}} \leqq H_{0}\left\|u_{y}-u\right\|_{\dot{R}},
$$

$\dot{R}$ and $\epsilon$ being such that all $R^{\prime}+y$ are in $\dot{R}$ and all $R+y$ are in $R$ when $y$ is in $C_{\text {e. }}$ Property IV then yields (5.7).

Relations (5.5) and (5.7) are sufficient to establish the properties of the mollifiers in the sense of $\$ 2$.

It is necessary to generalize inequality (5.5). Let $h_{\mathrm{e}}(y)$ be as before and let $g_{e}\left(x^{\prime}, \bar{x}\right)$ be a matrix which is continuous for $x^{\prime}$ in $R^{\prime}, \bar{x}$ in $R$. Then we

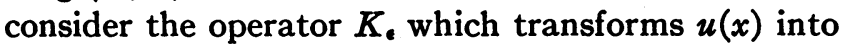

$$
K_{\epsilon} u\left(x^{\prime}\right)=\int_{R} h_{e}(\bar{x}-x) g_{\mathrm{e}}\left(x^{\prime}, \bar{x}\right) u(\bar{x}) d x .
$$

We set

With $(7)$

$$
\left\langle\left\langle g_{e}\right\rangle\right\rangle=\underset{x^{\prime}, \bar{x}}{\text { l.u.b. }}\left|g_{\epsilon}\left(x^{\prime}, \bar{x}\right)\right|, \quad \bar{x} \text { in } C_{\epsilon}\left(x^{\prime}\right), x^{\prime} \text { in } R^{\prime} .
$$

$$
\left\|K_{\mathrm{e}}\right\|=\left\|H_{\mathrm{e}}\right\|\left\langle\left\langle g_{\mathrm{e}}\right\rangle\right\rangle
$$

we then have, corresponding to (2.3),

$$
\left\|K_{\epsilon} u\right\|_{R^{\prime}} \leqq\left\|K_{\mathrm{e}}\right\|\|u\|_{R}
$$

To prove inequality $(5.8)$ we write $K_{6}$ in the form

$$
K_{\epsilon} u\left(x^{\prime}\right)=\int_{C_{\epsilon}} h(y) g_{\epsilon, y}\left(x^{\prime}\right) u_{y}\left(x^{\prime}\right) d y
$$

with

$$
g_{\epsilon, y}\left(x^{\prime}\right)=g_{\epsilon}\left(x^{\prime}, x^{\prime}+y\right) .
$$

By virtue of property III we have for $\boldsymbol{y}$ in $C_{\text {. }}$

$$
\left\|g_{\epsilon, y} u_{y}\right\|_{R^{\prime}} \leqq\left\langle g_{\epsilon, y}\right\rangle_{R^{\prime}}\left\|u_{y}\right\|_{R^{\prime}} \leqq\left\langle\left\langle g_{\epsilon}\right\rangle\right\rangle\left\|u_{y}\right\|_{R^{\prime}} .
$$

When now for $u$ in $\dot{\mathfrak{\varepsilon}}, K_{\epsilon} u\left(x^{\prime}\right)$ is approximated by

$$
\alpha g_{\epsilon, a}\left(x^{\prime}\right) u_{a}\left(x^{\prime}\right)+\beta g_{\epsilon, b}\left(x^{\prime}\right) u_{b}\left(x^{\prime}\right)+\cdots,
$$

(7) The norm (5.8) is somewhat weaker than the norm $\left\|K_{\mathrm{e}}\right\|$ employed in $\$ 2$; it is, however, sufficient for our purposes. 
we have

$$
\begin{aligned}
\left\|\alpha g_{\epsilon, a} u_{a}+\beta g_{\epsilon, b} u_{b}+\cdots\right\|_{R^{\prime}} & \leqq|\alpha|\left\|g_{\epsilon, a} u_{a}\right\|_{R^{\prime}}+|\beta|\left\|g_{\epsilon, b} u_{b}\right\|_{R^{\prime}}+\cdots \\
& \leqq\left\langle\left\langle g_{\epsilon}\right\rangle\right\rangle\left\{|\alpha|\left\|u_{a}\right\|_{R^{\prime}}+|\beta|\left\|u_{b}\right\|_{R^{\prime}}+\cdots\right\} \\
& \leqq\left\langle\left\langle g_{\epsilon}\right\rangle\right\rangle\{|\alpha|+|\beta|+\cdots\}\|u\|_{R^{\prime}}
\end{aligned}
$$

Thus, by a limit process as before, we obtain (5.9) first for $u$ in $\dot{夭}$, then for $u$ in $\mathfrak{R}$.

Inequality (5.9) is sufficient to derive relations $(2.6)_{0}$ for the special operators $K_{\epsilon}$ now considered. Clearly the same holds for the sum of such operators. The operator

$$
\bar{D}_{\mu}\left(A_{\mu}^{\prime}-\bar{A}_{\mu}\right) J_{\epsilon} \cdot=-\bar{D}_{\mu} \bar{A}_{\mu} \cdot J_{\epsilon}+\left(A_{\mu}^{\prime}-\bar{A}_{\mu}\right) \bar{D}_{\mu} J_{\epsilon} \cdot
$$

is such a sum. Indeed, $\left\langle\left\langle\bar{D}_{\mu} \bar{A}_{\mu}\right\rangle\right\rangle$ and $\left\|J_{\epsilon}\right\|$ are bounded; also $\epsilon^{-1}\left\langle\left\langle A_{\mu}^{\prime}-\bar{A}_{\mu}\right\rangle\right\rangle$ and $\epsilon\left\|\bar{D}_{\mu} J_{\epsilon} \cdot\right\|$ are bounded. Therefore, the argument of $\$ 3$ remains valid.

As a result the main theorem holds for norms of the general type considered in this section.

6. Miscellaneous applications. In this section we give divers applications of the main theorem.

TheOREM 6.1. Let $B^{(n)}, A_{\mu}^{(n)}$ be a sequence of matrices which together with the derivatives of $A^{(n)}$ converge to limit matrices $B, A_{\mu}$, uniformly in every proper subregion $\boldsymbol{R}^{\prime}$ of $\boldsymbol{R}$. Let $\mathfrak{F}^{(n)}=\mathfrak{F}\left(E^{(n)}\right)$ be the space $\mathfrak{F}$ with reference to the operator $E^{(n)}=A_{\mu}^{(n)} D_{\mu}+B^{(n)}$. Let $u$ and $v$ be functions in $\mathfrak{L}$, and let $u^{(n)}$ be a sequence in $\mathfrak{F}^{(n)}$ such that $\left\|u^{(n)}-u\right\|_{R^{\prime}} \rightarrow 0,\left\|E^{(n)} u^{(n)}-v\right\|_{R^{\prime}} \rightarrow 0$ for every proper $R^{\prime}$. Then $u$ is in $\mathfrak{F}$ and $E u=v$.

This theorem states that the strong definition could be weakened by permitting $E u$ to be approximated by $E^{(n)} u^{(n)}$ without widening the domain of applicability of $E$.

To prove it we need only observe that for every $u^{*}$ in $\mathfrak{D}$

$$
\left(E^{(n) *} u^{*}, u^{(n)}\right)_{R}=\left(u^{*}, E^{(n)} u^{(n)}\right)_{R} ;
$$

hence

$$
\left(E^{*} u^{*}, u^{(n)}\right)_{R}=\left(u^{*}, v\right)_{R},
$$

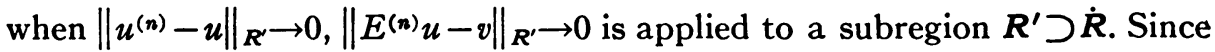
$u^{*}$ was arbitrary, $u$ is in $\mathcal{G}, E u=v$ and the main theorem yields the statement.

An application of a different character is the following. Let $E=A_{\mu} D_{\mu}+B$ be a differential operator with $A_{\mu}$ possessing second, $B$ first continuous derivatives. Let $O=P_{\mu} D_{\mu}+Q$ be an operator such that

$$
O E=0
$$

"formally," that is, $P_{\mu} A_{\nu}+P_{\nu} A_{\mu}=0, P_{\mu} D_{\mu} A_{\nu} \cdot+P_{\nu} B+Q A_{\nu}=0, P_{\nu} D_{\nu} B \cdot+Q B$ 
$=0$, in obvious notation. Then, clearly, for every $u$ in $\mathfrak{D}^{2}$, that is, possessing continuous second derivatives, $Q E u=0$. We now state

Theorem 6.2. For $u$ in $\mathfrak{F}(E), E u$ is in $\mathfrak{F}(O)$ and $O E u=0$.

Indeed, we have for $u^{*}$ in $\mathfrak{D}, u$ in $\mathfrak{D}^{2}$,

$$
0=\left(u^{*}, O E u\right)_{R}=\left(O^{*} u^{*}, E u\right)_{R} .
$$

By virtue of the strong definition of $\mathfrak{F}(E)$, relation $\left(O^{*} u, E u\right)_{R}=0$ remains valid for $u$ in $\mathfrak{F}(E)$ (since $J_{\epsilon} u$ is in $\mathfrak{D}^{2}$ ); by virtue of the weak definition of $(B)(O)$, it follows that $E u$ is in $\mathbb{S}(O)$ and $O E u=0$, but $\mathbb{S}(O)=\mathfrak{F}(O)$. As a corollary to Theorem 4.2 we state

TheOREM 6.3. Let w be in $\mathfrak{F}\left(O^{*}\right)$, then $O^{*} w$ is in $\mathfrak{F}\left(E^{*}\right)$ and $E^{*} O^{*} w=0$.

Relation $\left(O^{*} u^{*}, E w\right)=0$ is valid for $u^{*}$ in $\mathfrak{D}, u$ in $\mathfrak{F}(E)$, hence for $u$ in $\mathfrak{D}$, and for $u^{*}$ in $\mathfrak{F}\left(O^{*}\right)$. Therefore $O^{*} u$ is in $\left(\$\left(E^{*}\right), E^{*} O^{*} w=0\right.$, but $(\$)\left(E^{*}\right)=\mathfrak{F}\left(E^{*}\right)$.

If the operator $O^{*}=-P_{\mu}^{*} D_{\mu}+\left(Q^{*}-D_{\mu} A_{\mu}^{*} \cdot\right)$ were such that $A_{\mu}^{*}$ had continuous second, $Q^{*}-D_{\mu} A_{\mu}^{*} \cdot$ continuous first derivatives, Theorem 4.3 would follow from Theorem 4.2. It is to be noted that Theorem 4.3 holds without such differentiability conditions.

The significance of Theorem 6.3 is the following. Let

$$
E^{*} v=0
$$

be an underdetermined system $(s<t)$, then

$$
E u=z
$$

is an overdetermined system. There are necessary conditions on $z$ in $\mathbb{R}$ that $E u=z$ can have solutions $u$ in $\mathfrak{F}(E)$. Let $O z=0$ be such a condition with $O E=0$ formally, then

$$
v=O^{*} w, \quad w \text { in } \mathfrak{F}\left(O^{*}\right)
$$

represents a solution of $E^{*} v=0$.

More generally it could be shown that if $O_{1} \cdots O_{k} E=0$ formally, assuming appropriate differentiability conditions on the matrices involved, then

$$
v=O_{k}^{*} \cdots O_{1}^{*} w
$$

$w$ in $\mathfrak{F}$,

is a solution of $E^{*} v=0\left({ }^{8}\right)$.

As another application of the main theorem, or rather, of the arguments

(8) This simple procedure for obtaining "integral-free" solutions of underdetermined systems has apparently not been formulated in the literature. (Cf. for example P. Zervos, Le problème de Monge, Mémorial des Sciences Mathématiques, LIII (1932)). It may be mentioned that it can be used for a justification of Lagrange's multiplier method, when the side conditions are linear differential equations. 
that led to the main theorem, we prove a statement which is essentially equivalent to "Haar's lemma." It refers to a pair of functions $u=\left\{u_{1}, u_{2}\right\}$ of two variables $\left\{x_{1}, x_{2}\right\}$, and concerns the operators divergence, which transforms $u$ into

$$
\operatorname{div} u=D_{1} u_{1}+D_{2} u_{2},
$$

and rotation, which transforms a function $v$ into the system

$$
\operatorname{rot} v=\left\{D_{2} v,-D_{1} v\right\} \text {. }
$$

These operators are connected through the formal relation $\operatorname{div} \operatorname{rot} v=0$. The theorem then reads as follows.

THEOREM 6.4. Let $\boldsymbol{R}$ be a two-dimensional simply-connected region. Let $u=\left\{u_{1}, u_{2}\right\}$ be a function in (S)(div) such that div $u=0$. Then a function $v$ in $\mathfrak{F}$ (rot) exists such that

$$
u=\operatorname{rot} v \text {. }
$$

Proof. If $u$ belongs to $\mathfrak{D}$ then it is well known that a function $v$ in $\mathfrak{D}$ with $u=\operatorname{rot} v$ exists. The function $v$ is unique within an additive constant. We fix this constant by requiring

$$
\int_{C_{0}} v d x=0
$$

$C_{0}$ being a circle, properly contained in $R$, chosen at pleasure. Then through $v=V u$ a linear operator is defined for all $u$ in $\mathfrak{D}$ with div $u=0$; for such $u$, the relation rot $V u=0$ holds identically. We proceed to show that this operator $V$ can be extended to all $u$ in (S) (div) with div $u=0$. Let $u$ be such a function, $\boldsymbol{R}^{\prime}$ a proper subregion. Then we consider the sequence $u_{\epsilon}=J_{\boldsymbol{c}} u$, defined for any subregion $R^{\prime \prime}$. By virtue of identity (3.1) (for $E=$ div), we have $\operatorname{div} u_{\epsilon}=J_{\epsilon} \operatorname{div} u=0$. Hence the operator $V$ is applicable to $u_{\epsilon}$. We have $\left\|u_{\epsilon}-u\right\|_{R^{\prime \prime}} \rightarrow 0$ as $\epsilon \rightarrow 0$, or $\left\|u_{\epsilon}-u_{\delta}\right\|_{R^{\prime \prime}} \rightarrow 0$ as $\epsilon, \delta \rightarrow 0$. We now make use of the

LEMma. To every proper subregion $R^{\prime}$ there is a proper subregion $R^{\prime \prime} \subset R^{\prime}$ and a constant $C$ such that for $u$ in $\mathfrak{D}$ with $\operatorname{div} u=0$

$$
\|V u\|_{R^{\prime}} \leqq C\|u\|_{R^{\prime \prime}}
$$

We postpone the proof of this lemma. We observe that (6.3) implies $\left\|V u_{e}-V u_{\delta}\right\|_{R^{\prime}} \rightarrow 0$; hence there is a function $v$ in $\mathbb{R}$ such that $\left\|V u_{c}-v\right\|_{R^{\prime} \rightarrow 0}$. Since rot $V u_{\epsilon}=u_{\epsilon}$ and $\left\|u_{\epsilon}-u\right\|_{R^{\prime} \rightarrow 0}$ it is clear that $v$ is in $F$ (rot) and rot $v=u$.

Inequality (6.3) is, in view of (6.2), equivalent with

$$
\left\|v-v_{0}\right\|_{R^{\prime}} \leqq C\|\operatorname{rot} v\|_{R^{\prime \prime}}
$$

where 


$$
v_{0}=\int_{c_{0}} v d x / \int_{c_{0}} d x .
$$

Inequality (6.4) is a generalized form of Poincare's inequality; to prove it one need only combine the reasoning presented in Courant-Hilbert, vol. 2, chap. $7, \S 8$ for $p=2$ and rather general domains, with the reasoning of Morrey [14b] for $p \geqq 1$ and rectangular cells. One observes that every proper subregion of a simply connected region can be covered by a proper subregion $R^{\prime \prime}$ of the type $\mathfrak{N}$ considered in Courant-Hilbert at the indicated place.

\section{BIBLIOGRAPHY}

1. K. Friedrichs, On differential operators in Hilbert spaces, Amer. J. Math. vol. 61 (1939).

2. - On hyperbolic differential operators, Bull. Amer. Math. Soc. Abstract 46-9-416 (1940) p. 754.

3. M. Bobcher, On harmonic functions in two dimensions, Proceedings of the American Academy of Sciences vol. 41 (1905-1906).

4. G. C. Evans, On the reduction of integro-differential equations, Trans. Amer. Math. Soc. vol. 15 (1914) p. 485 . Note on a theorem of Bocher, Amer. J. Math. vol. 50 (1928) pp. 123-126.

5. - Fundamental points of potential theory, Rice Institute Pamphlets vol. 7 (1920) p. 274. Complements of potential theory. I, Amer. J. Math. vol. 55 (1933) p. 42.

6. C. B. Morrey, Jr., A class of representations of manifolds. I, Amer. J. Math. vol. 55 (1933) p. 687.

7. J. W. Calkin, Functions of several variables and absolute continuity. I, Duke Math. J. vol. 6 (1940) p. 274.

8. N. Wiener, The operational calculus, Math. Ann. vol. 95 (1926) p. 582.

9. R. Courant and D. Hilbert, Methoden der mathematischen Physik, vol. 2, p. 470.

10. D. C. Lewis, Infinite systems of ordinary differential equations, Trans. Amer. Math. Soc. vol. 35 (1933) p. 808.

11. H. Weyl, The method of orthogonal projection in potential theory, Duke Math. J. vol. 7 (1940) p. 411.

12. K. Ogura, Tôhoku Math. J. vol. 16 (1919).

13. J. von Neumann, Über adjungierte Funktionaloperatoren, Ann. of Math. vol. 33 (1932) p. 294.

14. C. B. Morrey, Jr., Functions of several variables and absolute continuity. II, Duke Math. J. vol. 6 (1940) p. 187. (a) Theorem 7.1. (b) Theorem 8.3.

15. - On the solutions of quasilinear elliptic partial differential equations, Trans. Amer. Math. Soc. vol. 43 (1938) p. 126. Existence and differentiability theorems for the solutions of variational problems for multiple integrals, Bull. Amer. Math. Soc. vol. 46 (1940) p. 439.

NEW YORK UNIVERSITY,

NEW YoRK, N. Y. 